\title{
Developing Prior Distributions for Bayesian Meta-Analyses
}

Kaitlan M. McKinney*, Angelika M. Stefan, \& Quentin F. Gronau Psychological Methods Department University of Amsterdam Amsterdam, the Netherlands

*Corresponding Email: kaitlan.mckinney@gmail.com 


\begin{abstract}
One effective way to consolidate studies is to use Bayesian meta-analyses. Bayesian methodology allows researchers to integrate their own predictions into the analysis. However, researchers may struggle to decide which prior distribution is appropriate. To help researchers in this pursuit, we outline a method to develop prior distributions based on past data. We constructed three prior distributions based on 100 social psychology meta-analyses of the past 20 years. We fit distributions to the data using maximum likelihood estimation. We then tested the effectiveness of our prior distributions against uninformed alternatives using testing data that had been separated out during the fitting process. Our prior distributions resulted in larger Bayes factors than the alternatives. Our results showcase the methodology's potential to develop informed prior distributions for various purposes in the future.
\end{abstract}

Keywords: Bayesian meta-analysis, prior distribution, prior specification, social psychology 


\section{Developing Prior Distributions for Bayesian Meta-Analyses}

The meta-analysis is a powerful tool in psychology research (Cooper \& Lemke, 1991). They allow researchers to integrate findings from multiple individual studies that investigate the same empirical phenomenon (Glass, 1976). Combining information from multiple studies has several advantages. Firstly, psychology researchers are often interested in small effects that are difficult to detect with small sample sizes (Gignac \& Szodorai, 2016, Maxwell, 2004). Since meta-analyses combine participants across studies, they can provide high-powered tests of hypotheses in the presence of small effects, even if individual studies are under-powered (Cohn \& Becker, 2003). Secondly, meta-analyses can be used to assess the variation of effect sizes between studies, also called effect size heterogeneity. Specifically, meta-analytic studies either utilize a fixed-effect model, assuming that all observed effect sizes in the sample stem from a single true underlying effect, or a random-effects model, assuming that the true underlying effect may differ from study to study (Borenstein et al., 2009). For example, researchers may expect variations in effect sizes due to variations in methodology between the studies. A third advantage of meta-analyses is that they allow researchers to investigate factors that may have contributed to between-study differences through moderator analyses (Borenstein \& Higgins, 2013). For example, if one half of the studies suggests a positive effect and the other half suggests a negative effect, meta-analyses can be used to test hypotheses about potential moderators that explain this divergence.

One way to conduct meta-analyses is to use Bayesian methods. Analogous to classical approaches (e.g., Borenstein \& Higgins, 2013), Bayesian meta-analyses compile data from existing studies, have the ability to detect smaller effects, and can examine the influence of moderators on study effect sizes (Gronau et al., 2020; Heck et al., 2019). However, the Bayesian approach presents several additional benefits for applied researchers. Among others, Bayesian meta-analyses enable researchers to quantify evidence in favor of both the 
alternative and the null hypothesis via the Bayes factor (Gronau et al., 2017; Quintana \& Williams, 2018). Importantly, meta-analytic evidence can be continuously updated as new studies roll in (Wagenmakers, Morey, \& Lee, 2016). Obtaining evidence in favor of the null hypothesis can prove especially important in light of the replication crisis, where many labs failed to reproduce published findings (Kvarven et al., 2020). In a Bayesian meta-analysis, these null results can be combined with information from original studies to test whether the overall data provide evidence for the null or alternative hypothesis.

Bayesian meta-analyses also allow researchers to incorporate knowledge about a phenomenon into the analysis using a prior distribution (Gelman, 2002; Sutton \& Abrams, 2001). A prior distribution is a probability distribution that reflects a researcher's beliefs about the plausibility of parameter values before seeing the data. For example, replication labs might use effect sizes obtained in the original study as prior knowledge to inform the prior distribution because they expect replication results to be similar to the original study (Ly et al., 2018). Additionally, if a researcher has extensive knowledge in the field, they may have precise expectations about effect sizes, and therefore can specify a narrow range of values for the prior distribution with the highest probability on the most likely ones (Stefan et al., 2020).

Informed prior distributions allow researchers to constrain model predictions to realistic values that are aligned with psychological theory and existing expert knowledge (Roever, 2017; Vanpaemel \& Lee, 2012). This is also the case for meta-analyses where different effect sizes and levels of heterogeneity can be expected depending on the scientific field and investigated research question. However, psychology researchers often experience difficulties in formulating informed prior distributions because they need to decide on a particular family of probability distributions as well as on the exact parameters of the distribution that are used to define its shape. For many statistical analyses, including meta-analyses, there are no clear 
guidelines for how to specify reasonable informed prior distributions. Therefore, researchers often resort to uninformed default distributions, such as a wide zero-centered Cauchy distribution on effect sizes (Morey \& Rouder, 2015), despite their typically lower diagnosticity.

Here, we propose a method for specifying informed prior distributions in the context of meta-analyses based on existing literature. In order to formulate an informed prior distribution for meta-analyses, researchers should first know how meta-analytic effect sizes are distributed in the field. To limit the scope of our paper, we will focus only on the subfield of social psychology. However, our method will also be applicable to other fields.

There have been several studies providing an overview of effect sizes in psychology. A recent study by Gignac and Szodorai (2016) examined 87 meta-analyses from several different fields in psychology and found a median effect size of $r=.19$. However, Gignac and Szodorai’s (2016) estimate was not specific to social psychology. Notably, a study by Richard and colleagues (2003) aggregated research exclusively in social psychology. The researchers identified every social psychology meta-analysis published until 1997; they identified 322 studies in total. The researchers found an overall meta-analytic effect size of $r$ $=.21$. As Richard and colleagues (2003) study is based on data that is more than 20 years old, we decided to update their original estimate of social psychology effect sizes with novel research from the past twenty years in order to develop a prior distribution. Compilations of meta-analytic data like Richard et al. (2003), Gignac and Szodorai (2016), and the present study provide an evidence-based estimate of the distribution of effect sizes in the respective fields, which can be a good starting point for an informed prior distribution. In this way, we outline a method that researchers can follow to develop priors for their own subfields.

While the main goal of our research is to generate and test a method for developing prior distributions, an additional benefit is a compilation of meta-analysis research across 
social psychology. The impact of our research is therefore three-fold: firstly, we describe a method to develop informed prior distributions for meta-analyses based on the example of social psychology; secondly, we demonstrate the impact of these informed priors on the results of a Bayesian meta-analytic hypothesis test compared to a default prior distribution; and thirdly, we provide a current compilation of meta-analytic effect sizes in social psychology.

\section{Prior Specification Method}

To develop a prior distribution for meta-analyses from existing literature, the first step is to compile a list of existing meta-analyses in the respective research field. Note that it is necessary for the collected studies to be of meta-analytic nature because only meta-analyses provide estimates for between-study heterogeneity. The study inclusion criteria determine how specific the prior distribution is with regard to the field and research question. For example, inclusion criteria can focus on a single effect (e.g., the bystander effect) or on a specific sub-field (e.g., prosocial behavior), or they can include all meta-analyses from a psychological discipline (e.g., social psychology). With increasing generality of inclusion criteria, more variation can be expected with respect to the reported quantities, which will be reflected in a greater variance of the prior distribution. In a second step, different families of distributions can be fit to the extracted meta-analytic effect size and heterogeneity estimates and a distribution can be selected that fits the data reasonably well. This prior distribution can then be applied to meta-analyses of new data in the field. Figure 1 provides an illustration of 
this prior specification process. In the following, we will demonstrate the prior specification process for our example of social psychology meta-analyses.

\section{Figure 1}

Visualization of Research Design.

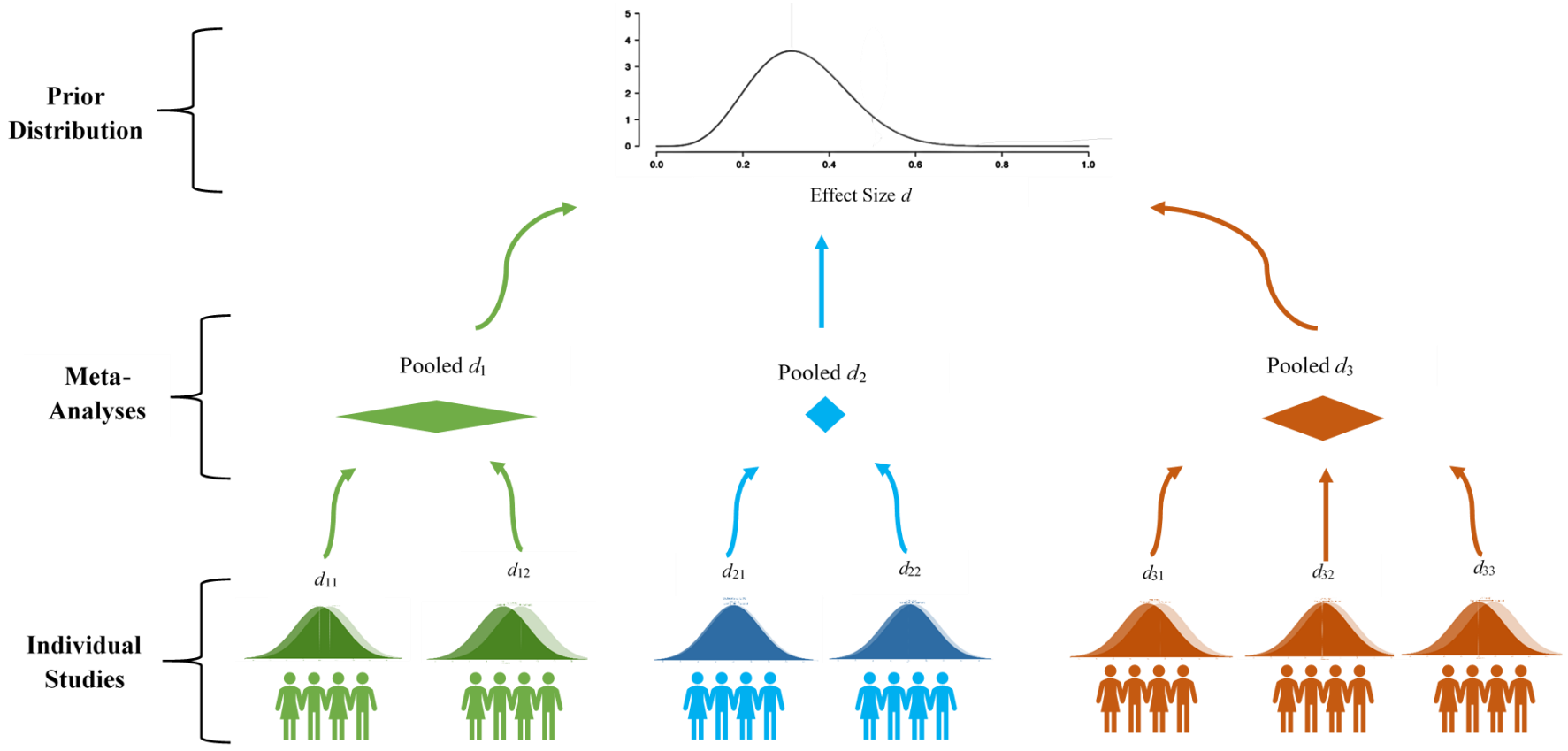

Note. Overview of the proposed process of formulating an informed prior distribution from meta-analyses. Meta-analyses (middle level) pool existing data from individual studies (bottom level). We pool data from these meta-analyses to create a prior distribution (top level). The effect size distributions were created using the Shiny widget created by Magnusson (2020).

\section{Study Inclusion Criteria}

In order to have enough data points to model a distribution, we planned to collect at least 50 effect sizes. For our focus on social psychology meta-analyses, we collected articles from Psychological Bulletin that were published between the years of 2000 and 2020. We chose Psychological Bulletin because the journal exclusively publishes synthesized research (e.g., meta-analyses), and is one of the leading journals to do so (https://www.apa.org/pubs/journals/bul). 
We used a set of inclusion criteria for selecting articles. Making a list helped us to limit the scope to only data that was relevant to the prior. To be included in our sample, studies needed to conduct a novel meta-analysis (rather than reanalyze an existing one) in the field of social psychology, specifically from one of the subfields listed in Supplementary Text S1. For each article, we consulted the list of subfields and noted in a table which subfield the study belongs to (see Table 1 for an example). In the table, we also noted the reason for exclusion if a study was related to social psychology but did not belong to one of these subfields. Noting down these decisions can allow multiple researchers to contribute simultaneously and also helps to keep the study transparent and reproducible. A table with all studies that were screened for the current manuscript, as well as their field categorization and inclusion/exclusion status can be found at https://osf.io/2xubf/?view_only=e6c79a85a3fc47abb6600e9b4bdd160e. 


\section{Table 1}

Coding document for included studies

\begin{tabular}{cc}
\hline Column Heading & Example \\
\hline Full Reference & $\begin{array}{c}\text { Zell, E., Strickhouser, J. E., Sedikides, C., \& Alicke, } \\
\text { M. D. (2020). The better-than-average effect in }\end{array}$ \\
& comparative self-evaluation: A comprehensive review \\
& and meta-analysis. Psychological Bulletin, 146, 118- \\
Year & 149. \\
Topic & 2020 \\
Subfield of Social Psychology & Attribution \\
Main Effect & Better-than-average effect \\
Type of Effect & personality traits as better than the average peer. \\
Fixed/Random & Cohen's d \\
Pooled Effect Estimate & Random \\
T5\% Confidence Interval - Lower & 0.78 \\
95\% Confidence Interval - Upper & 0.71 \\
Tau Squared & 0.84 \\
Number of studies & 0.29 \\
Total n & 291 \\
\hline & 950,000 \\
\hline &
\end{tabular}

We decided to record only one effect size estimate per study to avoid giving more weight to some studies than to others; therefore, we only collected the main effect.

Furthermore, as only a few studies investigated effects with a negative sign, we recoded these effects so that they were phrased in a positive way. For example, the effect "women are less likely to initiate negotiations than men" with a negative effect size was recoded to "men are more likely to initiate negotiations than women" with a positive effect size (Kugler et al., 2018). Additionally, if a study was a random-effects analysis, we also recorded a heterogeneity estimate, $\tau$. 


\section{Distribution Fitting Procedure}

In a second step, we fit probability distributions separately for the extracted metaanalytic effect size and heterogeneity estimates. We used R (R Core Team, 2020) for the distribution fitting. All code and data can be found on the Open Science Framework (https://osf.io/2xubf/?view_only=e6c79a85a3fc47abb6600e9b4bdd160e). We used the oldest $90 \%$ of the compiled studies for the distribution fitting procedure, leaving $10 \%$ of the data to investigate the results of Bayesian meta-analyses if the informed prior distributions were used. We fit a normal, a Cauchy, and a t-distribution (using the stats package) for the effect sizes. We fit an inverse gamma and a half-t distribution for the between-study heterogeneity. We chose these distributions as they are available in the statistics software JASP (JASP Team, 2020), and therefore more accessible to researchers. We us maximum likelihood estimation (using the fitdistr function from the MASS package) to determine which parameters were most appropriate for the data (for examples, see Myung, 2003). We used the Bayesian Information Criterion (BIC, Raftery, 1995; using the BIC function from the stats package) to select the best fitting model.

\section{Application of Prior Distributions in Bayesian Meta-Analyses}

We applied the specified prior distributions to Bayesian meta-analyses for all studies within the newest $10 \%$ of our study sample using Bayes factors calculated with the metaBMA package (Heck, 2019). We decided to test the prior distributions using the newest metaanalyses to simulate a situation where meta-analyses from the past inform future metaanalyses. To assess the impact of informed priors on Bayesian model comparisons, we compare the meta-analytic Bayes factor for our specified informed priors to meta-analytic Bayes factors obtained from uninformative default priors. 


\section{Results}

\section{Extracted Meta-Analytic Data}

Data were 100 meta-analyses and included every social psychology meta-analysis from the journal Psychological Bulletin volume 126, issue 1 (i.e., January, 2000), until volume 146, issue 6 (i.e., June, 2020; see Figure 2). We used the contained 33 correlation coefficients, 25 Cohen's d effect sizes, and 31 Hedge's g effect sizes. One study provided an estimate of heterogeneity but no estimate of a main effect.

\section{Figure 2}

\section{Exclusion Tree}

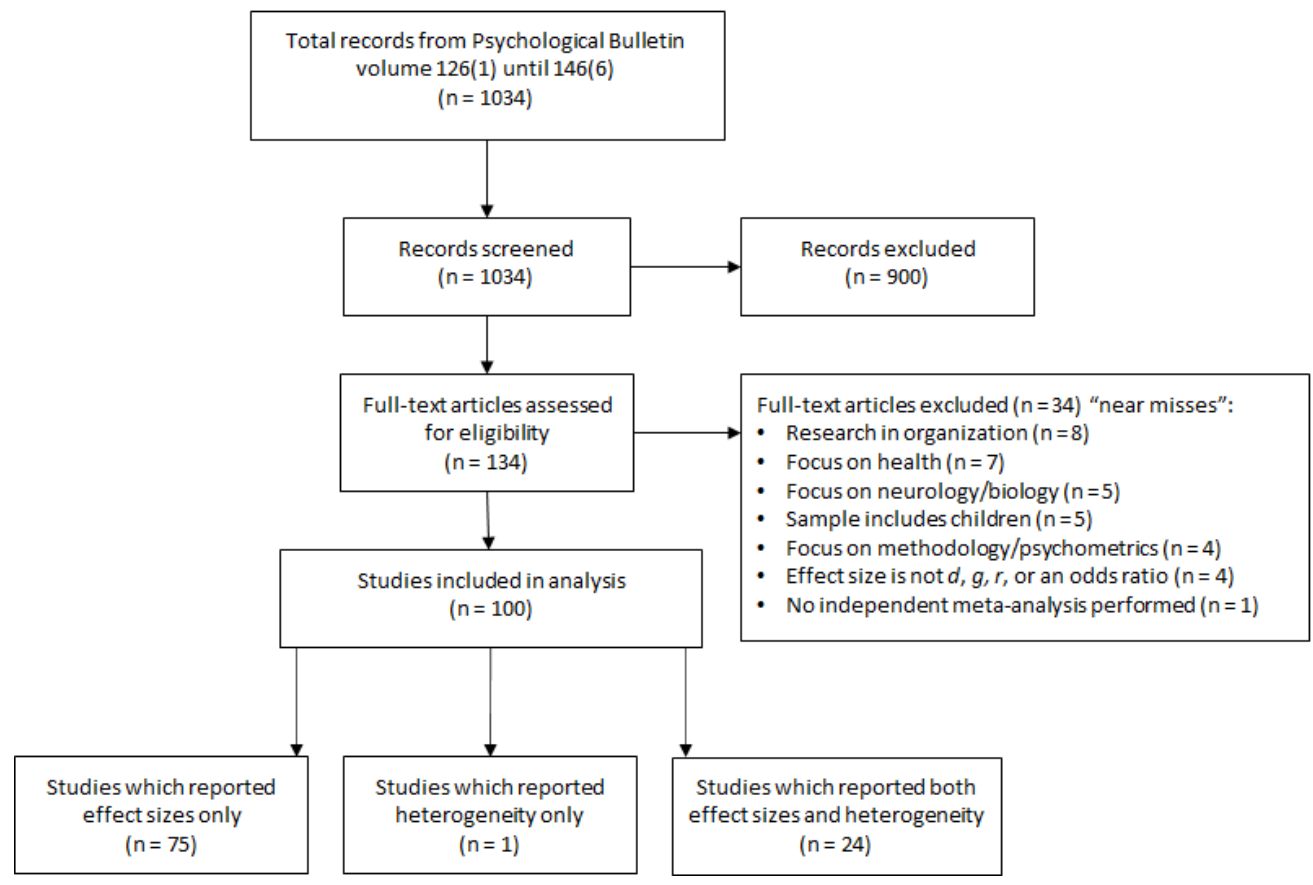

Note. Many of the studies were obviously unrelated to social psychology (i.e., 900 of them). We therefore only documented excluded studies when it was a "near-miss," and its inclusion could be debated.

Not every study provided an estimate of heterogeneity; in total there were 25 estimates of $\tau$.

We first examined the data for trends that could influence our prior specification results. Details on these exploratory analyses can be found in Supplementary Text S2.

Notably, we examined whether the correlation coefficients (CC) were on average numerically 
different from the mean difference effect sizes (MDES), and we found that they, in fact, did differ: After converting correlations to Cohen's $d$, a Bayesian $t$-test resulted in a Bayes factor of $\mathrm{BF}_{10}=278$, suggesting that the correlation coefficients and mean difference effect sizes differ on average.

\section{Prior Distributions for the Correlation Coefficients}

As CC are bounded between the values of -1 and 1, we converted the CC effect sizes to Fisher's $z$, which is unbounded. We used the following formula (from Borenstein et al., 2009, p. 42):

$$
z=.5 \times \log \frac{(1+r)}{(1-r)}
$$

We used maximum likelihood estimation to fit a truncated normal, truncated $t$, and truncated Cauchy distribution to the data. Truncation implies a directional hypothesis; we expected that researchers have knowledge about the direction of the effect, i.e., whether it is positive or negative. The following were the best-fitting distributions (see Figure 3): 
Figure 3

Prior Distributions Superimposed over Histogram of Fisher's z Effect Sizes

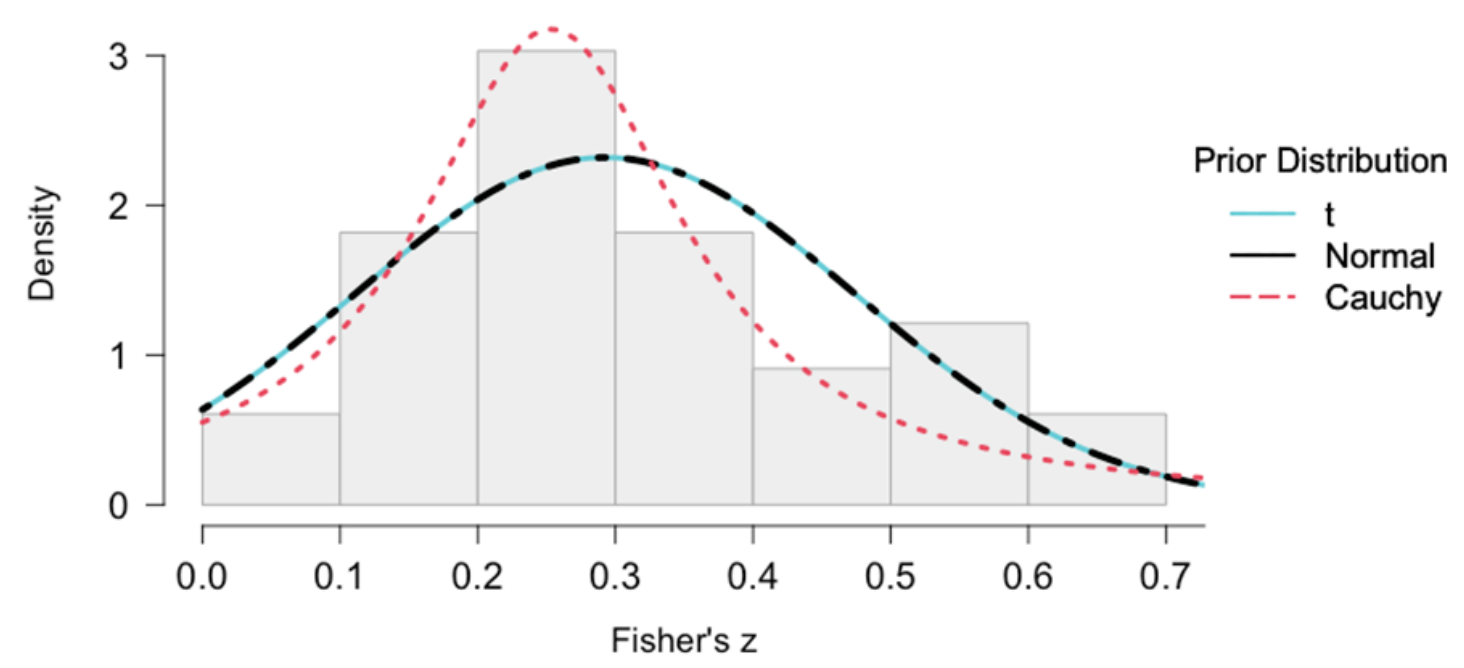

Note. The histogram in grey depicts the distribution of correlation coefficients in our social psychology study sample $(N=33)$. Correlation coefficients were transformed to Fisher's $z$. Curves represent the fitted prior distributions, which were truncated at zero. The $t$ distribution closely resembles a normal distribution. The normal distribution achieves the best fit to the data according to the BIC criterion.

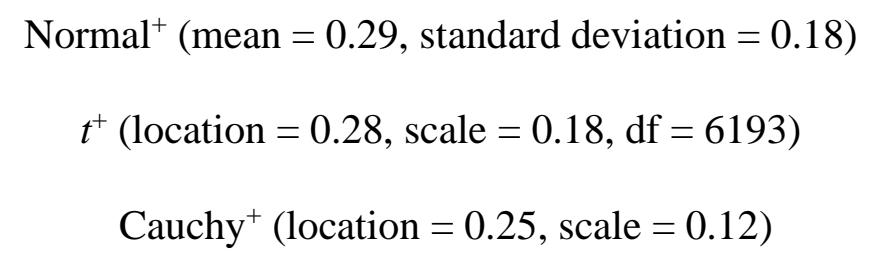

We used the BIC to determine which distribution fit the data the best. The BIC values were $-22,-18$, and -16 for the Normal, $t$, and Cauchy distribution, respectively. The Normal distribution had the lowest BIC, indicating the best fit of the three models, so we will use this prior in our comparison.

\section{Prior Distributions for the Mean Difference Effect Sizes}

We used maximum-likelihood estimation to fit a truncated normal, truncated $t$, and truncated Cauchy distribution to the effect sizes for mean differences extracted from the meta-analytic data. This resulted in the following parameters (see also Figure 4): 


\section{Figure 4}

Prior Distributions Superimposed over Histogram of Cohen's d and Hedge's g Effect Sizes

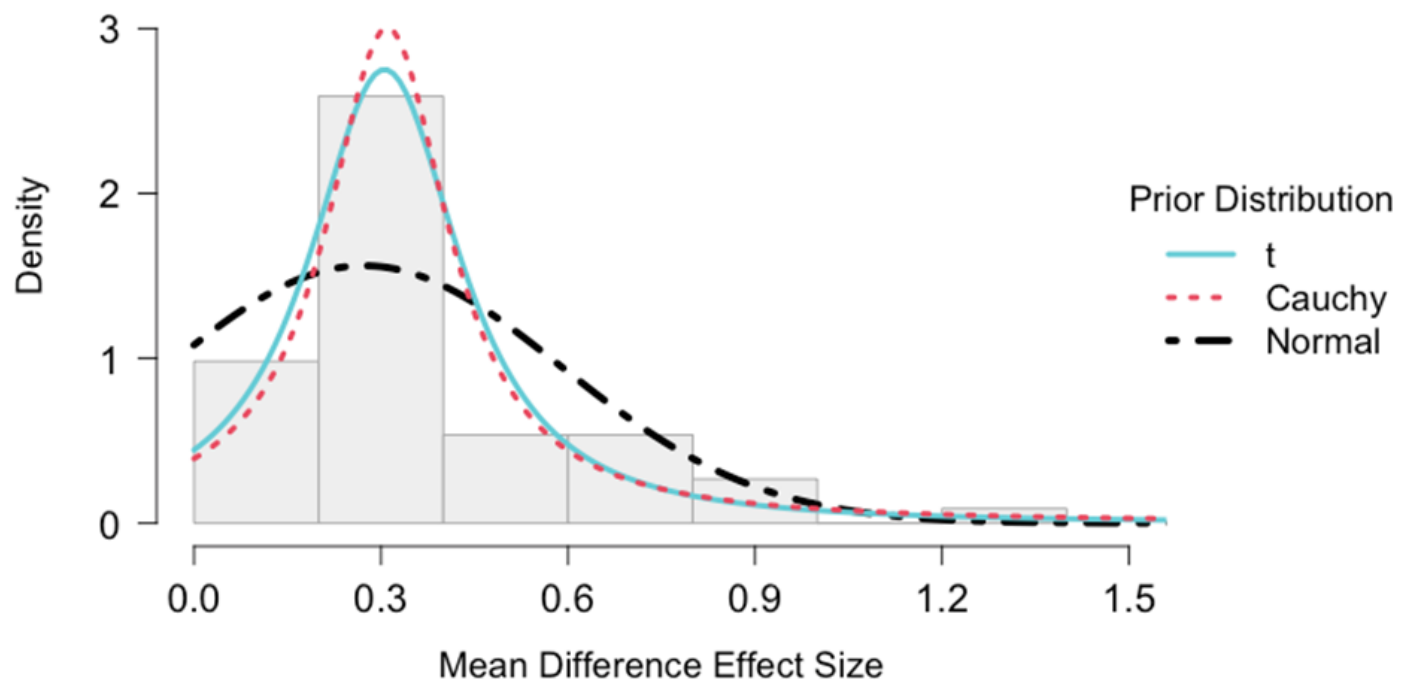

Note. The histogram in grey represents the data for the mean difference effect sizes $(N=$ 56), including Cohen's $d$ and Hedge's $g$. The curves represent the prior distributions based on this data, which were truncated at zero. The $t$ distribution closely resembles a Cauchy distribution.

$$
\begin{gathered}
\text { Normal }^{+}(\text {mean }=0.27, \text { standard deviation }=0.32) \\
t^{+}(\text {location }=0.31, \text { scale }=0.14, \mathrm{df}=1.4) \\
\text { Cauchy }^{+}(\text {location }=0.31, \text { scale }=0.12)
\end{gathered}
$$

The BIC values were $-2,-7$, and -10 for the Normal, $t$, and Cauchy distributions, respectively. The Cauchy distribution had the lowest BIC, and therefore it was the best fitting distribution for the MDES.

\section{Prior Distributions for Between-Study Heterogeneity}

Many studies reported conducting a random-effects meta-analysis but failed to report an estimate for the between-study heterogeneity coefficient $\tau$. Therefore, our sample for $\tau$ values only consisted of 25 estimates. We fit an inverse gamma distribution as well as a half- $t$ 
distribution to the empirical distribution of heterogeneity coefficients. A half- $t$ distribution resembles a truncated $t$-distribution, except that it is necessarily centered at zero. We obtained the following parameters:

$$
\begin{aligned}
& \text { Inverse Gamma }(\text { shape }=2.7, \text { scale }=0.5) \\
& \text { Half- } t(\text { standard deviation }=.29, \mathrm{df}=9053)
\end{aligned}
$$

As can be seen in Figure 5, neither distribution seems to fit the data very well. This is likely due to the small sample size for tau. The BIC values were -18.6 and -19.3 for the inverse gamma and half-t distribution, respectively. Therefore, the half-t distribution was a better fit for the data.

\section{Figure 5}

Prior Distributions for Tau Superimposed over the Histogram of Tau Data

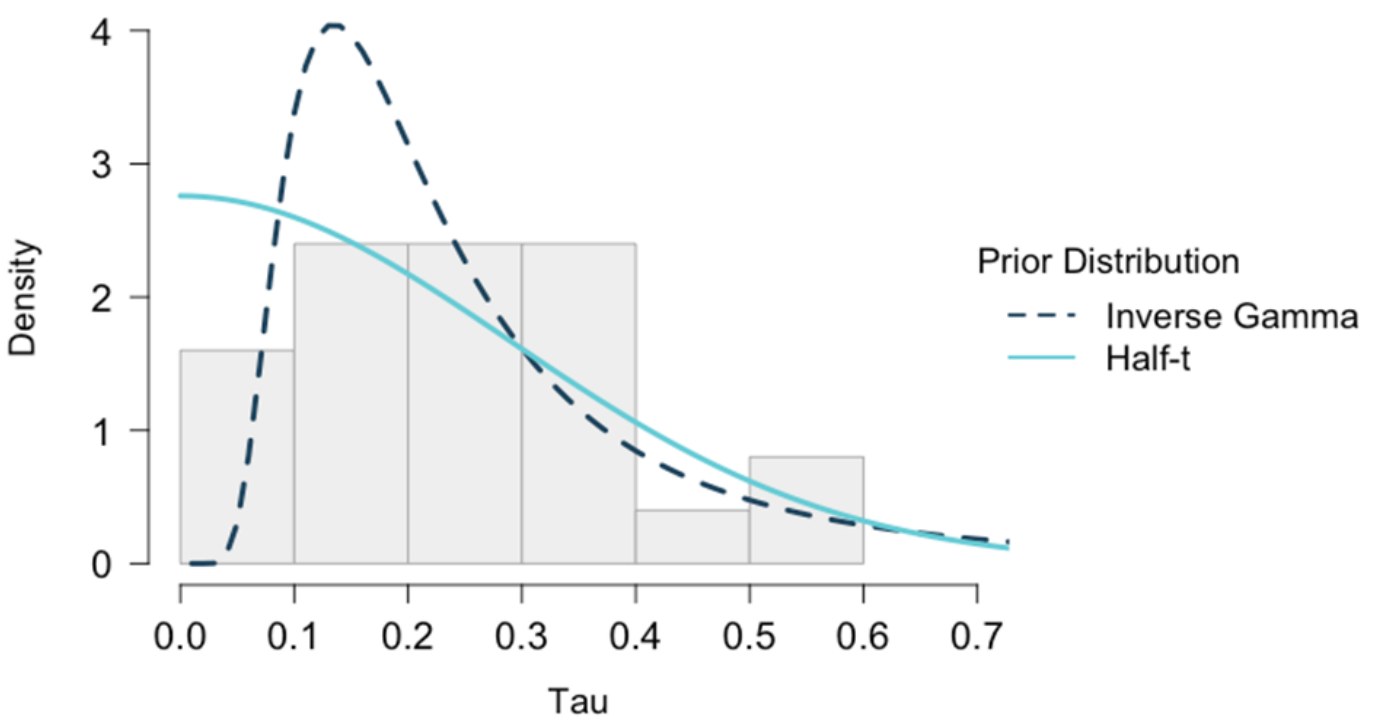

Note. The grey histogram represents the tau values from the learning data $(N=$ $25)$, while the curves represent the prior distributions we obtained for tau. 


\section{Meta-Analyses with Informed Prior Distributions}

We reanalyzed the most recently published $10 \%$ of the meta-analytic studies in our sample using Bayesian random-effects meta-analyses. Of the nine studies, four were investigating CCs and five were investigating MDES. We ran three Bayesian meta-analyses for each study (see Table 2): one using the best-fitting informed priors specified above, labeled I-1, and two using priors proposed as a default in the statistics software JASP, labeled D-1 and D-2, respectively (JASP Team, 2020).

\section{Table 2}

Prior distribution parameters for meta-analyses based on correlations and mean differences

Prior I-1

Prior D-1

Effect Size

Heterogeneity

Effect Size

Prior D-2

Heterogeneity
Prior Distribution Parameters

\begin{tabular}{cc}
\hline Correlations & Cohen's d \& Hedge's g \\
\hline Normal+ & Cauchy+
\end{tabular}

$($ mean $=0.29, \mathrm{sd}=0.18) \quad($ location $=.31$, scale $=0.12)$

Half-t

$(\mathrm{sd}=0.29, \mathrm{df}=9053)$

Half-t $(\mathrm{sd}=0.29, \mathrm{df}=9053)$

Normal+

Normal+ $($ mean $=0, \mathrm{sd}=0.707)$

Inverse gamma

$($ shape $=1$, scale $=0.15)$ $($ mean $=0, \mathrm{sd}=0.707)$

Inverse gamma

$($ shape $=1$, scale $=0.15)$

\section{Cauchy+}

Cauchy+ (location $=0$, scale $=0.707) \quad($ location $=0$, scale $=0.707)$ Inverse gamma $($ shape $=1$, scale $=0.15)$ ( shape $=1$, scale $=0.15)$

We conducted random-effects meta-analyses because it would allow us to incorporate both the prior on the effect size as well as the prior on tau (contrary to a fixed-effect metaanalysis, where tau is assumed to be zero). Estimates of the effect size, tau, and the Bayes factors under each of the three prior combinations can be found in Table 3 for studies based on correlations, and Table 4 for studies based on mean differences. The Bayes factors under 
the prior distribution that we developed (Prior 1) were larger than those under the other prior distributions in every case. 


\section{Table 3}

Estimates of Fisher's z, tau, and $B F_{10}$

Fisher's z $\quad$ Tau $\quad$ BF $_{10}$

\begin{tabular}{|c|c|c|c|c|c|c|c|c|c|c|}
\hline Meta-Analysis & $K$ & $\mathrm{I}-1$ & D-1 & D-2 & $\mathrm{I}-1$ & D-1 & D-2 & $\mathrm{I}-1$ & D-1 & D-2 \\
\hline Thielmann et al., 2020 & 56 & 0.105 & 0.104 & 0.104 & 0.066 & 0.067 & 0.068 & $5.24 \times 10^{7}$ & $4.74 \times 10^{7}$ & $3.74 \times 10^{7}$ \\
\hline Lui \& Quezada, 2019 & 72 & 0.199 & 0.199 & 0.198 & 0.115 & 0.114 & 0.114 & $3.81 \times 10^{17}$ & $3.27 \times 10^{17}$ & $2.52 \times 10^{17}$ \\
\hline Montoya et al., 2018 & 253 & 0.206 & 0.205 & 0.205 & 0.130 & 0.129 & 0.129 & $13.5 \times 10^{37}$ & $10.6 \times 10^{37}$ & $8.13 \times 10^{37}$ \\
\hline Cheng et al., 2019 & 706 & 0.086 & 0.086 & 0.087 & 0.197 & 0.198 & 0.197 & $7.92 \times 10^{22}$ & $7.67 \times 10^{22}$ & $6.08 \times 10^{22}$ \\
\hline
\end{tabular}

Note. All priors were truncated on the interval $[0, \infty]$. The parameters for each prior are listed in Table $2 . K$ represents the number of studies included in the meta-analysis.

The Bayes Factor $\mathrm{BF}_{10}$ is the ratio of the likelihood of obtaining the observed results under $\mathrm{H}_{1}$ to the likelihood of obtaining them under $\mathrm{H}_{0}$.

\section{Table 4}

Estimates of Cohen's d, tau, and $B F_{10}$
Cohen's $d$
Tau
$\mathrm{BF}_{10}$

Meta-Analysis $\quad \mathrm{K}$

I-1

D-1

Th-1

\begin{tabular}{|c|c|c|c|c|c|c|c|c|c|c|}
\hline Nitschke et al., 2019 & 20 & 0.371 & 0.388 & 0.388 & 0.334 & 0.332 & 0.333 & 6593 & 2649 & 1890 \\
\hline Dargue et al., 2019 & 83 & 0.607 & 0.625 & 0.623 & 0.448 & 0.456 & 0.454 & $31.8 \times 10^{13}$ & $11.5 \times 10^{13}$ & $7.61 \times 10^{13}$ \\
\hline Coles et al., 2019 & 286 & 0.234 & 0.227 & 0.226 & 0.405 & 0.405 & 0.405 & $19.4 \times 10^{11}$ & $9.31 \times 10^{11}$ & $7.09 \times 10^{11}$ \\
\hline Zell et al., 2020 & 291 & 0.773 & 0.774 & 0.776 & 0.537 & 0.539 & 0.539 & $29.3 \times 10^{67}$ & $7.46 \times 10^{67}$ & $4.94 \times 10^{67}$ \\
\hline Asperholm et al., 2019 & 821 & 0.176 & 0.176 & 0.175 & 0.385 & 0.385 & 0.385 & $13.9 \times 10^{27}$ & $11.4 \times 10^{27 \#}$ & $8.87 \times 10^{27} \# \#$ \\
\hline
\end{tabular}

The Bayes Factor $\mathrm{BF}_{10}$ refers to the ratio of the likelihood of obtaining the observed results under $\mathrm{H}_{1}$ to the likelihood of obtaining them under $\mathrm{H}_{0}$

\# This Bayes Factor is based on a model with $\hat{R}=1.015$, and therefore may be unreliable.

\#\# This Bayes Factor is based on a model with $\hat{R}=1.013$, and therefore may be unreliable. 


\section{Discussion}

\section{Main Findings}

The main goal of our research was to develop a methodology that researchers can use to specify prior distributions for Bayesian meta-analyses. As a proof of concept, we applied the methodology to derive informed prior distributions for effect sizes and between-study heterogeneity for meta-analyses in the field of social psychology. We tested our prior distributions on nine recently published meta-analyses and found that our priors resulted in larger Bayes factors than the default, uninformed priors from JASP in every case. This shows that the informed priors robustly improved diagnosticity of the tests. The informed priors outperformed the default priors for small effect sizes (i.e., Fisher's $z=0.086$ ), medium effect sizes (i.e., Cohen's $d=0.371$ and $d=0.607$ ), and large effect sizes (i.e., Cohen's $d=0.773$ ).

Our secondary goal was to compare our results to the study of social psychology meta-analyses by Richard and colleagues (2003) to determine whether the field has changed drastically over the past 20 years. We hypothesized that effect sizes could have changed as methods adapt, researchers' focus shifts to more niche topics, and the field morphs and divides. Interestingly, the effect size distribution in our data is very similar to that of 20 years ago. For example, Richard and colleagues (2003) reported an overall effect size of $r=.18$, while the median of our study (across the different effect sizes) is $r=.19$. This suggests that effect sizes have remained relatively stable over the years. This finding is notable because, at least for social psychology, we can predict that the distribution of effect sizes will likely remain similar in the future, as well. This also suggests that researchers do not have to rely solely on current research to inform their Bayesian analyses, but can also rely on results from studies in the past.

With regards to the between-study heterogeneity, our search yielded only 25 estimates of tau. This was enough for us to create a distribution. However, with such a small sample 
size, the validity of the distribution parameters can be compromised. To obtain a robust prior distribution, it might thus be necessary to extend the range of included studies. For example, Van Erp and colleagues (2017) used a similar methodology to retrieve 705 heterogeneity estimates from meta-analyses across 23 years. The researchers also used Psychological Bulletin to retrieve meta-analyses, although their study was not exclusive to social psychology. These parameters developed by van Erp and colleagues (2017) are likely much more reliable, as the sample size is far larger, but the resulting prior distribution would no longer be specific to social psychology.

Although our method produces well-informed prior distributions, the method itself is quite time consuming. Researchers who wish to follow this method must spend a substantial amount of time finding articles and extracting effect sizes from the literature. It would not be feasible to use this methodology before every single study. However, once specified, these prior distributions can be reused for other meta-analyses in the same (sub)field. This opens the possibility to community-based solutions to the prior specification problem, such as openaccess repositories of prior distributions. In addition to the time-saving effects of sharing data publicly, another benefit of repositories is that data can be filtered and reanalyzed. For example, researchers could filter the dataset to include only effect sizes that relate to their study topic, resulting in a prior distribution which is more specific. We want to practice what we preach; therefore, we have published our data and code, so researchers can filter and reanalyze these data as they see fit. In sum, although the method is time consuming, the data that is collected could be used to generate both general prior distributions for the field as a whole as well as filtered to estimate more study-specific prior distributions.

Furthermore, the method that we have outlined is advantageous because it produces prior distributions which are specific to a particular field, in this case social psychology, yet general enough to be applied to any meta-analysis in the field. The resulting prior could be 
thought of as a default informed prior for the field. Another way to choose a prior would be to ask an expert to estimate the distribution. One downside of asking experts is that the prior distribution needs to be determined separately for each research question. Often this expertelicited prior will be far too specific to be used for any other study. If the researcher is unsure of the size of the (meta-analytic) effect, then they could rely on the data-driven prior distributions that our method produces because they are much more general.

One concern about this method is the longevity of the prior distributions it produces. As research practices change for the better, and researchers become more aware of publication bias, average effect sizes may change over time. However, as our distribution of effect sizes resembled that of Richard et al. (2003) from nearly 20 years ago, we do not expect the change in the future to be very drastic. In any case, our prior distributions seem to be quite robust despite the magnitude of the effect size, and therefore will likely perform well in the future despite changes to the field.

Another point of consideration is the degree to which the Bayes factors change as the prior distribution changes. As an example, consider the study by Zell et al. (2020): We used the same data and analyses across the board, but the Bayes factor under Prior I-1 was five times larger than the Bayes factor under Prior D-2 (see Table 4). Considering these differences, one might wonder whether prior distributions can make the findings seem more conclusive than they really are. Here, we argue that incorporating historic prior knowledge is not a way of "hacking" the Bayes factor, but that the informed prior leads to a more diagnostic test of theory (Vanpaemel \& Lee, 2012). The higher Bayes factor is the reward for constraining the model predictions by allocating more prior mass to a smaller range of values that are deemed plausible a-priori. While using informed priors often leads to higher Bayes factors, it rarely alters the substantive conclusion of the hypothesis test (Wagenmakers et al., 2018; Stefan et al., 2021). Indeed, the substantive conclusion for the study by Zell et al. 
(2020) is the same under both Prior I-1 and Prior D-2: there is decisive evidence for an effect. With sufficient data, the prior distribution also has little effect on the posterior distribution. This means that effect size estimates based on models with different prior distributions differ only slightly, as can be seen in Table 3 and 4 of this paper.

In practice, many researchers are not only interested in main effects, but also in subsequent moderation analyses to explain influential factors. As we developed our prior distributions using main effects, these priors may not be suitable for moderation analyses. We recommend that researchers who wish to perform a meta-analysis with moderators first use our prior distributions to establish whether the effect is present and estimate the size of the main effect, and then choose a new prior distribution based on their own hypotheses to use for the moderation analysis.

Thus far, we have outlined both the benefits and limitations of the method we used to develop prior distributions. We strongly recommend branching out to examine possible prior distributions for meta-analyses for other fields in psychology. Not only would it be theoretically interesting to see how similar or different the fields of psychology are, but it would also be practically useful. A prior distribution represents our knowledge about a phenomenon, and therefore compiling data and developing prior distributions can help future researchers to make more specific predictions. Meta-analytic prior distributions could also serve as a starting point for developing study-specific priors for single studies within a field. For example, we found that social psychology effect sizes center around about $d=0.3$; if a researcher wanted to conduct a study on prosocial effects, they might expect an effect size that is slightly larger than other studies in social psychology and thus could use a prior centered at 0.35 instead. In theory, researchers could use the more general "field" prior and think about whether their effect size will rather fall in the higher or lower range of this prior and adjust it accordingly so that it fits their specific research scenario. 
In conclusion, we have outlined a method that researchers can use to determine informed prior distributions. We tested our methodology and developed prior distributions informed by 20 years of meta-analysis research. Finally, we propose a call for research into informed prior distributions for other fields of psychology, so that Bayesian statistics might be more accessible for everyone. 


\section{References}

* References marked with an asterisk indicate studies used as test data in the analysis.

Alston, C., Kuhnert, P., Low Choy, S., McVinish, R., \& Mengersen, K. (2005). Bayesian model comparison: Review and discussion. In 55th Session of the International Statistical Institute. https://www.stat.auckland.ac.nz/ iase/publications/13/Alston$\underline{\text { Kuhnert-Low_Choy-McVinish-Mengersen.pdf }}$

* Asperholm, M., Hogman, N., Rafi, J., \& Herlitz A. (2019). What did you do yesterday? A meta-analysis of sex differences in episodic memory. Psychological Bulletin, 145, 785821. https://doi.org/10.1037/bul0000197Association for Psychological Science. (n.d.). Registered replication reports. https://www.psychologicalscience.org/publications/replication

Borenstein, M., Hedges, L. V., Higgins, J. P. T., \& Rothstein, H. (2009). An Introduction to Meta-Analysis. Wiley. https://doi.org/10.1002/9780470743386.

Borenstein, M., \& Higgins, J. P. T. (2013). Meta-analysis and subgroups. Prevention Science, 14, 134-143. https://doi.org/10.1007/s11121-013-0377-7

* Cheng, C., Wang, H. Y., Sigerson, L., \& Chau, C. I. (2019). Do the socially rich get richer? A nuanced perspective on social network site use and online social capital accrual. Psychological Bulletin, 145, 734-764. http://dx.doi.org/10.1037/bul0000198

Cohen, J. (1988). Statistical power analysis for the behavioral sciences. Second Edition. Hillsdale, NJ: Lawrence Erlbaum Associates, Publishers.

Cohn, L. D., \& Becker, B. J. (2003). How meta-analysis increases statistical power. Psychological Methods, 8, 243-253. https://doi.org/10.1037/1082-989X.8.3.243

* Coles, N. A., Larsen, J. T., \& Lench, H. C. (2019). A meta-analysis of the facial feedback literature: Effects of facial feedback on emotional experience are small and variable. Psychological Bulletin, 145, 610-651. https://doi.org/10.1037/bul0000194 
Cooper, H. M., \& Lemke, K. M. (1991). On the role of meta-analysis in personality and social psychology. Personality and Social Psychology Bulletin, 17, 245-251. https://doi.org/10.1177/0146167291173002

* Dargue, N., Sweller, N., \& Jones, M. P. (2019). When our hands help us understand: A meta-analysis into the effects of gesture on comprehension. Psychological Bulletin, 145, 765-784. https://doi.org/10.1037/bul0000202

Gelman, A. (2002). Prior distribution. In Encyclopedia of Environmetrics. (Vol. 3, pp. 1634 1637). Chichester, England: John Wiley \& Sons, Ltd.

Gignac, G. E., \& Szodorai, E. T. (2016). Effect size guidelines for individual differences researchers. Personality and Individual Differences, 102, 74-78. https://doi.org/10.1016/j.paid.2016.06.069

Glass, G. V. (1976). Primary, Secondary, and Meta-Analysis of Research. Educational Researcher, 5, 3-8. https://doi.org/10.2307/1174772

Gronau, Q. F., Heck, D. W., Berkhout, S. W., Haaf, J. M., \& Wagenmakers, E. (2020). A Primer on Bayesian Model-Averaged Meta-Analysis. PsyArXiv Preprint https://doi.org/10.31234/osf.io/97qup

Gronau, Q. F., van Erp, S., Heck, D. W., Cesario, J., Jonas, K. J., \& Wagenmakers, E.-J. (2017). A Bayesian model-averaged meta-analysis of the power pose effect with informed and default priors: the case of felt power. Comprehensive Results in Social Psychology, 2, 123-138. https://doi.org/10.1080/23743603.2017.1326760

Heck, D. W. (2019). metaBMA: Bayesian model averaging for random and fixed effects meta-analysis. [R package version 0.6.2.] https://CRAN.Rproject.org/package $=$ metaBMA

Heck, D. W., Gronau, Q. F., \& Wagenmakers, E.-J. (2019). metaBMA: Bayesian model averaging for random and fixed effects meta-analysis [Computer software manual]. 
Retrieved from https://CRAN.R-project.org/package=metaBMA (R package version $0.6 .1)$

JASP Team (2020). JASP (Version 0.14.1) [Computer software]. https://jasp-stats.org/ Joyce, D. (2016). “Common probability distributions." https://mathcs.clarku.edu/ djoyce/ma218/distributions.pdf

Kugler, K. G., Reif, J. A. M., Kaschner, T., \& Brodbeck, F. C. (2018). Gender differences in the initiation of negotiations: A meta-analysis. Psychological Bulletin, 144, 198-222. https://doi.org/10.1037/bul0000135

Kvarven, A., Strømland, E., \& Johannesson, M. (2020). Comparing meta-analyses and preregistered multiple-laboratory replication projects. Nature Human Behaviour, 4, 423-434. https://doi.org/10.1038/s41562-019-0787-z

Lee, M.D., \& Wagenmakers, E.-J. (2013). Bayesian cognitive modeling: A practical course. Cambridge University Press.

* Lui, P. P., \& Quezada, L. (2019). Associations between microaggression and adjustment outcomes: A meta-analytic and narrative review. Psychological Bulletin, 145, 45-78. https://doi.org/10.1037/bul0000172

Ly, A., Etz, A., Marsman, M., \& Wagenmakers, E.-J. (2019). Replication Bayes factors from evidence updating. Behavior Research Methods, 51(6), 2498-2508. https://doi.org/10.3758/s13428-018-1092-x

Magnusson, K. (2020, February 22). Interpreting Cohen's d effect size: An interactive visualization. R Psychologist. https://rpsychologist.com/d3/cohend/

Maxwell, S. E. (2004). The persistence of underpowered studies in psychological research: Causes, consequences, and remedies. Psychological Methods, 9, 147-163. https://doi.org/10.1037/1082-989X.9.2.147 
Maxwell, S. E., Lau, M. Y., \& Howard, G. S. (2015). Is psychology suffering from a replication crisis? What does "failure to replicate" really mean? American Psychologist, 70, 487-498. https://doi.org/10.1037/a0039400

* Montoya, R. M., Kershaw, C., \& Prosser, J. L. (2018). A meta-analytic investigation of the relation between interpersonal attraction and enacted behavior. Psychological Bulletin, 144, 673-709. https://doi.org/10.1037/bu10000148

Morey, R. D., \& Rouder, J. N. (2015). BayesFactor: Computation of Bayes Factors for Common Designs. R package version 0.9.12-2. https://CRAN.Rproject.org/package $=$ BayesFactor

Myung, J. (2003). Tutorial on maximum likelihood estimation. Journal of Mathematical Psychology, 47, 90 - 100. https://doi.org/10.1016/S0022-2496(02)00028-7

* Nitschke, F. T., McKimmie, B. M., \& Vanman, E. J. (2019). A meta-analysis of the emotional victim effect for female adult rape complaints: Does complaint distress influence credibility? Psychological Bulletin, 145, 953-979. https://doi.org/10.1037/bul0000206

Plous, S. (n.d.). Social psychology links by subtopic. Social Psychology Network. https://www.socialpsychology.org/social.htm

"Psychological Bulletin," (n.d.). https://www.apa.org/pubs/journals/bul

Quintana, D. S., \& Williams, D. R. (2018). Bayesian alternatives for common null-hypothesis significance tests in psychiatry: A non-technical guide using JASP. BMC Psychiatry, 18. https://doi.org/10.1186/s12888-018-1761-4

R Core Team (2020). R: A language and environment for statistical computing. Vienna, Austria: R Foundation for Statistical Computing. https://www.R-project.org/ 
Raftery, A. E. (1995). Bayesian model selection in social research. In P. V. Marsden (Ed.), Sociological methodology (pp. 111-196). Cambridge, MA: Blackwell. https://doi.org/10.2307/271063

Richard, F. D., Bond, C. F. Jr., \& Stokes-Zoota, J. J. (2003). One hundred years of social psychology quantitatively described. Review of General Psychology, 7, 331-363. https://doi.org/10.1037/1089-2680.7.4.331

Roever, C. (2017). Bayesian random-effects meta-analysis using the bayesmeta R package. arXiv preprint 1711.08683 (submitted for publication).

Stefan, A. M., Evans, N. J., \& Wagenmakers, E.-J. (2020). Practical challenges and methodological flexibility in prior elicitation. Psychological Methods. Advance online publication. https://doi.org/10.1037/met0000354

Stefan, A., Katsimpokis, D., Gronau, Q. F., \& Wagenmakers, E.-J. (2021). Expert agreement in prior elicitation and its effects on Bayesian inference. PsyArXiv Preprint. $\underline{\text { https://doi.org/10.31234/osf.io/8xkqd }}$

Sullivan, G. M., \& Feinn, R. (2012). Using effect size - or why the $p$ value is not enough. Journal of Graduate Medical Education, 4, 279-282. https://doi.org/10.4300/JGME-D$\underline{12-00156.1}$

Sutton, A. J., \& Abrams, K. R. (2001). Bayesian methods in meta-analysis and evidence synthesis. Statistical Methods in Medical Research, 10, 277-303. https://doi.org/10.1177/096228020101000404

* Thielmann, I., Spadaro, G., \& Balliet, D. (2020). Personality and prosocial behavior: A theoretical framework and meta-analysis. Psychological Bulletin, 146, 30-90. https://doi.org/10.1037/bul0000217 
Vanpaemel, W., \& Lee, M. D. (2012). Using priors to formalize theory: Optimal attention and the generalized context model. Psychonomic Bulletin \& Review, 19, 1047-1056. https://doi.org/10.3758/s13423-012-0300-4

Wagenmakers, E.-J., Marsman, M., Jamil, T., Ly, A., Verhagen, J., Love, J., Selker, R., Gronau, Q. F., Smira, M., Epskamp, S., Matzke, D., Rouder, J. N., \& Morey, R. D. (2018). Bayesian inference for psychology. Part I: Theoretical advantages and practical ramifications. Psychonomic Bulletin \& Review 25, 35-57.

https://doi.org/10.3758/s13423-017-1343-3

Wagenmakers, E.-J., Morey, R. D., \& Lee, M. D. (2016). Bayesian benefits for the pragmatic researcher. Current Directions in Psychological Science, 25, 169-176.

https://doi.org/10.1177/0963721416643289

* Zell, E., Strickhouser, J. E., Sedikides, C., \& Alicke, M. D. (2020). The better-than-average effect in comparative self-evaluation: A comprehensive review and meta-analysis. Psychological Bulletin, 146, 118-149. https://doi.org/10.1037/bul0000218 


\section{Data Accessibility Statement}

All data and analysis scripts can be found on this paper's project page on the OSF repository, https://osf.io/2xubf/?view_only=e6c79a85a3fc47abb6600e9b4bdd160e . 